\title{
Microwave plasma emerging technologies for chemical processes
}

DOI:

$10.1002 / j c t b .5205$

\section{Document Version}

Accepted author manuscript

Link to publication record in Manchester Research Explorer

\section{Citation for published version (APA):}

de la Fuente, J. F., Kiss, A. A., Radoiu, M. T., \& Stefanidis, G. D. (2017). Microwave plasma emerging technologies for chemical processes. Journal of Chemical Technology and Biotechnology, 92(10), 2495-2505. https://doi.org/10.1002/jctb.5205

\section{Published in:}

Journal of Chemical Technology and Biotechnology

\section{Citing this paper}

Please note that where the full-text provided on Manchester Research Explorer is the Author Accepted Manuscript or Proof version this may differ from the final Published version. If citing, it is advised that you check and use the publisher's definitive version.

\section{General rights}

Copyright and moral rights for the publications made accessible in the Research Explorer are retained by the authors and/or other copyright owners and it is a condition of accessing publications that users recognise and abide by the legal requirements associated with these rights.

\section{Takedown policy}

If you believe that this document breaches copyright please refer to the University of Manchester's Takedown Procedures [http://man.ac.uk/04Y6Bo] or contact uml.scholarlycommunications@manchester.ac.uk providing relevant details, so we can investigate your claim.

\section{OPEN ACCESS}




\title{
Microwave plasma emerging technologies for chemical processes
}

\author{
Javier F. de la Fuente, ${ }^{1}$ Anton A. Kiss, ${ }^{2,3}$ Marilena T. Radoiu, ${ }^{4}$ Georgios D. Stefanidis ${ }^{5^{*}}$ \\ ${ }^{1}$ Process \& Energy Laboratory, Delft University of Technology, Leeghwaterstraat 39, 2628 \\ CB, Delft, The Netherlands, E-mail: J.FernandezdelaFuente@tudelft.nl \\ ${ }^{2}$ AkzoNobel - Supply Chain, Research \& Development, Process Technology SRG, \\ Zutphenseweg 10, 7418 AJ Deventer, The Netherlands. E-mail: Tony.Kiss@akzonobel.com \\ ${ }^{3}$ Sustainable Process Technology Group, Faculty of Science and Technology, University of \\ Twente, PO Box 217, 7500 AE, Enschede, The Netherlands \\ ${ }^{4}$ SAIREM SAS, 12 Porte du Grand Lyon, Neyron, 01702 Cedex France \\ (at present, this author is an independent consultant) \\ ${ }^{5}$ Chemical Engineering Department, Katholieke Universiteit Leuven, Celestijnenlaan 200f, \\ 3001 Leuven (Heverlee), Belgium
}

\begin{abstract}
Microwave plasma (MWP) technology is currently being used in application fields such as semiconductor and material processing, diamond film deposition and waste remediation. Specific advantages of the technology include the enablement of a high energy density source and a highly reactive medium, the operational flexibility, the fast response time to inlet variations and the low maintenance costs. These aspects make MWP a promising alternative technology to conventional thermal chemical reactors provided that certain technical and operational challenges related to scalability are overcome. Herein, an overview of state-ofthe-art applications of MWP in chemical processing is presented (e.g. stripping of photo resist, UV-disinfection, waste gas treatment, plasma reforming, methane coupling to olefins, coal/biomass/waste pyrolysis/gasification and $\mathrm{CO}_{2}$ conversion). In addition, two potential approaches to tackle scalability limitations are described, namely the development of a single unit microwave generator with high output power $(>100 \mathrm{~kW})$, and the coupling of multiple microwave generators with a single reactor chamber. Finally, the fundamental and engineering challenges to enable profitable implementation of the MWP technology at large scale are discussed.
\end{abstract}

Keywords: microwave plasma, emerging technologies, industrial applications, roadmap 


\section{1. Introduction}

2 A sustainable and green economy represents one of the major challenges of contemporary society. It involves mostly the reduction of waste generation, but also the optimization of raw material consumption in order to mitigate current alarming pollution problems and lower the energy requirements of industrial conversion processes. In this regard, the process intensification (PI) philosophy ${ }^{1}$ has significantly improved such industrial conversion processes. By applying the PI principles, ${ }^{2}$ various processing advances can be attained such as 1) higher energy efficiency, 2) lower raw material usage, 3) prevention of waste generation (improved product quality) and 4) safer process, as demonstrated in several industrial applications. ${ }^{3-5}$ For the chemical industry to progress towards a sustainable economy, novel waste-to-product approaches need to be developed to reduce the dependency on fossil fuels-based raw materials. Carbon Capture \& Utilization (CCU) is an emerging concept, which utilizes waste (e.g. greenhouse gases) as chemical feedstock to produce valuable products. ${ }^{6}$ In most cases however, the required energy input to transform waste into products tends to be rather high, making the re-utilization process unprofitable. Renewable energy sources, such as wind and solar power, are expected to have an increasing share in the future energy scene as a large fraction of the energy needed for chemical conversion processes can be obtained from these sources during peak electricity production periods. This falls within the so-called power-to-chemicals approach, ${ }^{7}$ whereby greenhouse gases and/or water are converted into hydrocarbons by means of surplus electric power.

In the context of power-to-chemicals approach, a large variety of electricity-based chemical reactors, such as electrolyzers, ${ }^{8}$ electrocatalytic reactors ${ }^{9}$ and plasma-based reactors ${ }^{10,11}$ have been investigated. The first two reactor types require the use of a catalyst, which potentially introduces problems of thermal degradation and coking at high temperatures. ${ }^{12}$ Concerning the use of electrolyzers, even though this technology has reached rather high voltage efficiencies (solid oxide electrolyzers, 80\%), ${ }^{13}$ the specific energy consumption compared to conventional hydrogen production routes (mainly steam reforming) is still relatively high. Plasma reactors represent a novel alternative technology due to certain processing benefits such as: fast process dynamics, process flexibility, no need for catalyst use in many processes, no need for (bulky and costly) gas-fired furnaces, low maintenance cost, and high quality products (low by-products formation). The performance of these three reactor types for the production of hydrogen was evaluated by Dincer et al. (2015). ${ }^{13}$ From the cost and energy efficiency point-of-view, they concluded that the plasma-assisted reactor performs better compared to the other electricity-based reactors. In this work, we focus on microwave plasma (MWP)-assisted reactors, which appear to be one of the most promising plasma reactor types as discussed hereafter. Plasma can be triggered and sustained by different energy sources. MWP in particular is a gas ionized by means of a high frequency $(300 \mathrm{MHz}-300 \mathrm{GHz})$ electromagnetic field. The alternating electric field heats the electrons which provide energy mostly for ionization and excitation of neutral atoms 
discharge types is that it does not require electrodes, which must be placed inside the reactor leading to operational issues, such as regular maintenance (replacement) due to erosion caused by contaminants formation. MWP can also be operated in a wide pressure range (from $10^{-5} \mathrm{mbar}-1$ bar). Furthermore, the power-to-plasma efficiency can reach up to $90 \% .{ }^{14}$ Concerning the reactor performance, the high frequencies at which MWP systems are operated produce a much larger fraction of electrons, i.e. higher electron densities, and higher electron temperature (energy) compared to other plasma sources. This results in high concentrations of active species rendering MWP an ideal highly reactive medium for chemical reactions. To date, most research has been carried out at laboratory-scale, while few successful industrial applications have been demonstrated. Some of the limitations that have hindered widespread implementation of MWP at industrial scale include the cost of equipment, challenges related to process scalability, controllability and stability as well as the cost of electric power. For further information on theoretical concepts related to MWP, the reader is referred to the literature. ${ }^{14-19}$ In this work, the state-of-the-art of MWP at laboratory stage, existing industrial chemical applications, current technical and operational limitations and an overview of the fundamental and engineering challenges for further development of the MWP technology are presented. Finally, some promising potential applications, which mainly concern high temperature processes, such as pyrolysis, gasification and reforming of organic waste, biomass and fossil fuels are discussed. Overall, this paper intends to set up a roadmap describing the main requirements and the next steps needed for the implementation of the MWP technology in the chemical manufacturing industry.

\section{Microwave plasma technology: state-of-the-art}

In this section, the necessary elements to operate MWP reactors are briefly described. Additionally, an overview of the laboratory state-of-the-art applications of MWP in chemical processing is given. Lastly, well-known industrial applications are discussed, showing the potential of the technology for commercial use.

\subsection{Description of the main elements}

In conventional chemical reactors, the design is characterized by process variables needing control during operation, such as temperature, pressure, concentrations of chemical species and throughput. With regard to MWP reactors, the waveguide component $\mathrm{s}^{20}$ are predefined and optimized for each particular operating frequency, whereas elements such as reactor sizing/shape/material, reactant inlet(s) (flow pattern) and cooling/control systems are design-related aspects that can be adjusted for optimization of the reactor performance. Table 1 and Figure 1 introduce the essential components of MWP systems. The component number 1 described in Table 1 (Microwave generator) is identified as the key element to scale up this type of reactors due to its limitation as to the maximum output microwave power. The reader is referred to the microwave generator section for further information. 
1 Table 1. Description of the main elements enabling the operation of MWP reactors

\begin{tabular}{|c|c|c|}
\hline $\mathbf{N}^{0}$ & Element & Function \\
\hline 1 & $\begin{array}{c}\text { Microwave generator } \\
= \\
\text { HV Power supply } \\
\text { (switch mode) \& } \\
\text { Microwave head }\end{array}$ & $\begin{array}{l}\text { Generation of electromagnetic waves } \\
\text { Operating frequency } 2.45 \mathrm{GHz} \text { : Maximum output power of } 15 \mathrm{~kW} \\
\text { Operating frequency } 915 \mathrm{MHz} \text { : Maximum output power of } 100 \mathrm{~kW}\end{array}$ \\
\hline 2 & Isolator & Protection of the magnetron from the reflected microwave field \\
\hline 3 & Impedance tuner & $\begin{array}{l}\text { Reduction of the reflected microwave power ensuring high energy transfer } \\
\text { efficiency from the microwave generator to the plasma zone; manual or } \\
\text { automatic operation }\end{array}$ \\
\hline 4 & Reactor assembly & $\begin{array}{l}\text { Conversion of raw materials into products } \\
\text { A plasma column is generated perpendicular to the incoming microwave field }\end{array}$ \\
\hline 5 & Movable plunger & $\begin{array}{l}\text { It positions the electromagnetic wave such that a local electric field maximum is } \\
\text { located at the center of the plasma zone to maximize the microwave energy } \\
\text { transfer from the generator to the plasma zone; manual or automatic operation }\end{array}$ \\
\hline
\end{tabular}

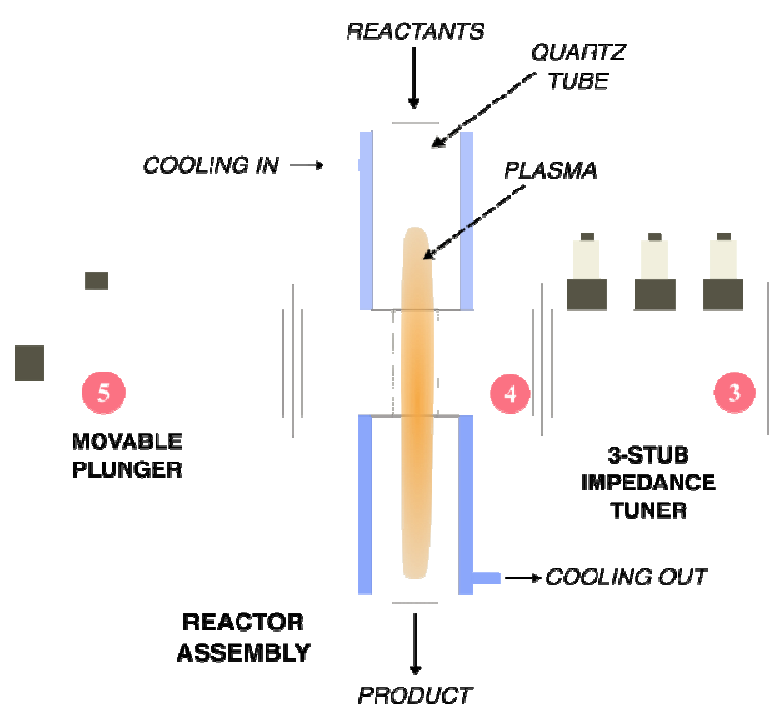

$2.45 \mathrm{GHz}$
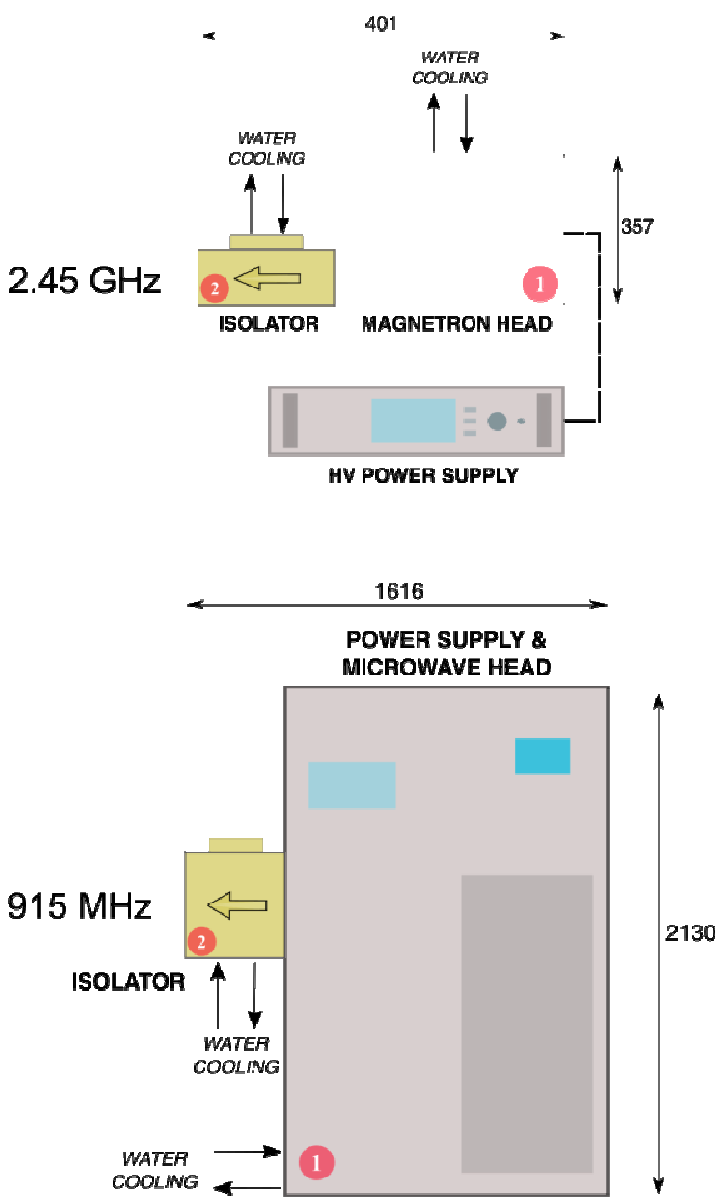

Figure 1. Sketch of the main elements needed to operate MWP reactors. Note that the two commercially available microwave generators for plasma applications $(2.45 \mathrm{GHz}-15 \mathrm{~kW}$ and 915 $\mathrm{MHz}-100 \mathrm{~kW}$ ) are outlined including the relevant dimensions $(\mathrm{mm})$; both generators consist of switch-mode HV power supplies. Circled numbers refer to the element numbers in Table 1; in this example, manually operated 3-stub tuner (3) and manually operated movable plunger (5). 


\subsection{State-of-the-art at laboratory stage}

3 A remarkably broad range of chemical applications at laboratory-scale have been explored in different

4 MWP systems with the exception of biomass gasification for syngas production, which has been investigated both at laboratory scale ${ }^{21}$ and in medium-scale plants. ${ }^{22,}{ }^{23}$ Concerning chemical manufacturing applications, several processes are highlighted due to the large number of research studies (see Table 2), such as: plasma-assisted reforming, biomass gasification and pyrolysis, $\mathrm{H}_{2}$ production, and $\mathrm{CO}_{2}$ utilization. We should note that the MWP technology is also used for other applications, including: production of carbon nanostructures of high quality (nanotubes, graphene), production of synthetic diamond for gemstones and technical applications, surface modification for deposition of thin films to improve material properties, water purification (ozone/UV radiation), photoresist stripping for removal of polymeric photoresists in semiconductor manufacturing, and medicine (cancer treatment, sterilization of medical instruments and disinfection of living tissue). Table 2 presents an overview of MWP-assisted processes for chemical applications. It is noticeable that the research focus has been on the production of synthesis gas or hydrogen. Herein, certain processes should be acknowledged due to the notably good performance shown in MWP reactors. As reported by Jasinski et al., ${ }^{24,}{ }^{25} \mathrm{H}_{2}$ generation via plasma-assisted dry reforming of methane can be carried out at a specific energy consumption of $\sim 3 \mathrm{kWh} / \mathrm{m}^{3} \mathrm{H}_{2}$ and a $\mathrm{H}_{2}$ generation cost $\sim 2.3 \$ / \mathrm{kg} \mathrm{H} \mathrm{H}_{2}$ (assuming $0.06 \$ / \mathrm{kWh}$ ). This value is close, within a factor 2 , to the reported industrial cost of $\mathrm{H}_{2}$ production (representative value: $\left.1.5 \$ / \mathrm{kg} \mathrm{H}_{2}\right)^{26,27}$ through the mature and highly integrated and optimized process of steam reforming of natural gas in high temperature furnaces. Another example is reported by Uhm et al. (2014), ${ }^{22}$ in which the gasification of brown coal in a pilot-scale setup was studied, obtaining cold gas efficiency (CGE, calorific power of produced synthetic gas divided by the total input power to the system) values up to $84 \%$, while conventional gasifiers, more specifically entrained flow gasifiers, have CGE values between $70-80 \% .{ }^{28}$ Additionally, one should take into account the evident gain in economy of scale and particularly the increase in the energy efficiency when microwave power above $1 \mathrm{~kW}$ is used to sustain the plasma chemical reactions, as displayed in Table 2. 
Table 2. Summary of reported MWP processes for chemical applications.

\begin{tabular}{|c|c|c|c|c|c|c|c|c|c|}
\hline $\begin{array}{l}\text { REACTANT } \\
\text { MIXTURE }\end{array}$ & $\begin{array}{l}\text { DESIREID } \\
\text { PRODUCT }\end{array}$ & $\begin{array}{l}\text { REACTOR } \\
\text { TYPE }\end{array}$ & PRESSURE & $\begin{array}{c}\text { POWER } \\
(\mathrm{kW})\end{array}$ & $\begin{array}{l}\text { FLOW RATE/ } \\
\text { THROUGHPUT }\end{array}$ & CONVERSION & SELECTIVITY (\%) & $\begin{array}{c}\text { ENERGY } \\
\text { EFFICIENCY } \\
\end{array}$ & REF. \\
\hline \multicolumn{10}{|c|}{ PLASMA ASSISTED REFORMING } \\
\hline $\mathrm{CH}_{4}+\mathrm{H}_{2} \mathrm{O}_{\mathrm{g}}$ & Syngas & $2.45 \mathrm{GHz}$ & 1 bar & $5(3-5)$ & $27 \mathrm{l} / \mathrm{min} \mathrm{H}_{2} \mathrm{O}_{\mathrm{g}}+3-9 \mathrm{l} / \mathrm{min} \mathrm{CH}_{4}$ & $95 \%$ & $70\left(\mathrm{H}_{2}\right) \& 19(\mathrm{CO})$ & 3001 syngas $/ \mathrm{kWh}$ & 29 \\
\hline $\mathrm{CH}_{4}+\mathrm{CO}_{2}$ & $\mathrm{H}_{2}$ & $2.45 \mathrm{GHz}$ & 1 bar & 4 & $60001 / \mathrm{h} \mathrm{CH}_{4}+6000 \mathrm{l} / \mathrm{h} \mathrm{CO}_{2}$ & $1100 \mathrm{l} / \mathrm{h} \mathrm{H}_{2}$ & - & $3501 \mathrm{H}_{2} / \mathrm{kWh}$ & 25 \\
\hline $\mathrm{C}_{2} \mathrm{H}_{5} \mathrm{OH}+\mathrm{N}_{2}$ & $\mathrm{H}_{2}$ & $915 \mathrm{MHz}$ & 1 bar & 5 & $\begin{array}{c}2700-39001 / \mathrm{h} \mathrm{N}_{2} \\
0.4-1.2 \mathrm{~kg} / \mathrm{h} \mathrm{C}_{2} \mathrm{H}_{5} \mathrm{OH}\end{array}$ & $1150 \mathrm{l} / \mathrm{h} \mathrm{H}$ & $100\left(\mathrm{H}_{2}\right)$ & $2671 \mathrm{H}_{2} / \mathrm{kWh}$ & 30 \\
\hline $\mathrm{C}_{3} \mathrm{H}_{7} \mathrm{OH}+\mathrm{CO}_{2}$ & $\mathrm{H}_{2}$ & $915 \mathrm{MHz}$ & 1 bar & 5 & $\begin{array}{c}2700 \text { 1/h CO } \\
2.4 \mathrm{~kg} / \mathrm{h} \mathrm{C}_{3} \mathrm{H}_{7} \mathrm{OH}\end{array}$ & $1116 \mathrm{l} / \mathrm{h} \mathrm{H} \mathrm{H}_{2}$ & $37\left(\mathrm{H}_{2}\right)$ & $2231 \mathrm{H}_{2} / \mathrm{kWh}$ & 30 \\
\hline $\mathrm{C}_{6} \mathrm{H}_{14}+\mathrm{H}_{2} \mathrm{O}_{\mathrm{g}}+\mathrm{Ar}$ & Syngas & $2.45 \mathrm{GHz}$ & 1 bar & 2 & $\begin{array}{c}34.6 \mathrm{~mol} / \mathrm{h} \mathrm{H}_{2} \mathrm{O}_{\mathrm{g}} \\
5 \mathrm{~mol} / \mathrm{h} \mathrm{C}_{6} \mathrm{H}_{14}\end{array}$ & $\begin{array}{c}36 \mathrm{~mol} / \mathrm{h} \mathrm{H} \mathrm{H}_{2} \\
14.4 \mathrm{~mol} / \mathrm{h} \mathrm{CO}\end{array}$ & - & $\begin{array}{c}0.24 \mathrm{~kg} \\
\text { syngas/kWh }\end{array}$ & 31 \\
\hline $\mathrm{C}_{8} \mathrm{H}_{18}+\mathrm{H}_{2} \mathrm{O}_{\mathrm{g}}+\mathrm{Ar}$ & Syngas & $2.45 \mathrm{GHz}$ & $1 \mathrm{bar}$ & 2 & $\begin{array}{l}34.6 \mathrm{~mol} / \mathrm{h} \mathrm{H} \mathrm{H}_{2} \mathrm{O}_{\mathrm{g}} \\
4.3 \mathrm{~mol} / \mathrm{h} \mathrm{C} \mathrm{H}_{18}\end{array}$ & $\begin{array}{l}21.6 \mathrm{~mol} / \mathrm{h} \mathrm{H} \mathrm{h}_{2} \\
10.8 \mathrm{~mol} / \mathrm{h} \mathrm{CO}\end{array}$ & - & $\begin{array}{c}0.17 \mathrm{~kg} \\
\text { syngas } / \mathrm{kWh}\end{array}$ & 31 \\
\hline $\mathrm{C}_{2} \mathrm{H}_{6}+\mathrm{Ar}$ & $\mathrm{C}_{2} \mathrm{H}_{4}$ & $2.45 \mathrm{GHz}$ & $20-73$ mbar & $2(0.1-0.8)$ & - & $95 \%$ & $50\left(\mathrm{C}_{2} \mathrm{H}_{4}\right)$ & - & 32 \\
\hline $\mathrm{C}_{5} \mathrm{H}_{12}+\mathrm{Ar}$ & $\mathrm{C}_{2} \mathrm{H}_{4}$ & $2.45 \mathrm{GHz}$ & $0.1 \mathrm{mbar}$ & $2(0.45)$ & $25 \mathrm{ml} / \mathrm{min} \mathrm{Ar}+7 \mathrm{ml} / \mathrm{h} \mathrm{C}_{5} \mathrm{H}_{12}$ & $65 \%$ & $40\left(\mathrm{C}_{2} \mathrm{H}_{4}\right)$ & $0.44 \mathrm{~g} \mathrm{C}_{2} \mathrm{H}_{4} / \mathrm{kWh}$ & 33 \\
\hline $\mathrm{C}_{6} \mathrm{H}_{14}+\mathrm{Ar}$ & $\mathrm{C}_{2} \mathrm{H}_{4}$ & $2.45 \mathrm{GHz}$ & $0.1 \mathrm{mbar}$ & $2(0.45)$ & $25 \mathrm{ml} / \mathrm{min} \mathrm{Ar}+7 \mathrm{ml} / \mathrm{h} \mathrm{C}_{6} \mathrm{H}_{14}$ & $72 \%$ & $65\left(\mathrm{C}_{2} \mathrm{H}_{4}\right)$ & $0.75 \mathrm{~g} \mathrm{C}_{2} \mathrm{H}_{4} / \mathrm{kWh}$ & 33 \\
\hline $\mathrm{C}_{7} \mathrm{H}_{16}+\mathrm{Ar}$ & $\mathrm{C}_{2} \mathrm{H}_{4}$ & $2.45 \mathrm{GHz}$ & $0.1 \mathrm{mbar}$ & $2(0.45)$ & $25 \mathrm{ml} / \mathrm{min} \mathrm{Ar}+7 \mathrm{ml} / \mathrm{h} \mathrm{C}_{7} \mathrm{H}_{16}$ & $75 \%$ & $55\left(\mathrm{C}_{2} \mathrm{H}_{4}\right)$ & $0.56 \mathrm{~g} \mathrm{C}_{2} \mathrm{H}_{4} / \mathrm{kWh}$ & 33 \\
\hline $\mathrm{C}_{8} \mathrm{H}_{18}+\mathrm{O}_{2}+\mathrm{Ar}$ & Syngas & $2.45 \mathrm{GHz}$ & 1 bar & 0.10 & $0.2 \mathrm{ml} / \mathrm{min} \mathrm{C}_{8} \mathrm{H}_{18}+7 \mathrm{lpm} \mathrm{Ar}$ & $68.4 \mathrm{~g} / \mathrm{h}$ syngas & - & $\begin{array}{c}0.68 \mathrm{~kg} \\
\text { syngas } / \mathrm{kWh} \\
\end{array}$ & 34 \\
\hline Gasoline $+\mathrm{O}_{2}+\mathrm{Ar}$ & Syngas & $2.45 \mathrm{GHz}$ & 1 bar & 0.10 & $\begin{array}{c}0.2 \mathrm{ml} / \mathrm{min} \text { gasoline }+ \\
7 \mathrm{lpm} \mathrm{Ar}\end{array}$ & $84.6 \mathrm{~g} / \mathrm{h}$ syngas & - & $\begin{array}{c}0.85 \mathrm{~kg} \\
\text { syngas } / \mathrm{kWh}\end{array}$ & 34 \\
\hline $\mathrm{CH}_{4}+\mathrm{O}_{2}+\mathrm{Ar}$ & Syngas & $2.45 \mathrm{GHz}$ & 1 bar & 0.10 & $140 \mathrm{ml} / \mathrm{min} \mathrm{CH}_{4}+7 \mathrm{l} / \mathrm{min} \mathrm{Ar}$ & $46.8 \mathrm{~g} / \mathrm{h}$ syngas & - & $\begin{array}{c}0.47 \mathrm{~kg} \\
\text { syngas } / \mathrm{kWh} \\
\end{array}$ & 34 \\
\hline $\mathrm{CH}_{4}+\mathrm{O}_{2}$ & $\mathrm{CH}_{3} \mathrm{OH}$ & $2.45 \mathrm{GHz}$ & $31 \mathrm{mbar}$ & 0.02 & $\begin{array}{c}30 \mathrm{ml} / \mathrm{min} \mathrm{CH}_{4}+ \\
50 \mathrm{ml} / \mathrm{min} \mathrm{O}_{2}\end{array}$ & $97 \%$ & $4.4\left(\mathrm{CH}_{3} \mathrm{OH}\right)$ & $\begin{array}{c}1.3 \mathrm{~kg} \\
\mathrm{CH}_{3} \mathrm{OH} / \mathrm{kWh} \\
\end{array}$ & 35 \\
\hline \multicolumn{10}{|c|}{ METHANE COUPLING TO OLEFINS } \\
\hline $\mathrm{CH}_{4}$ & $\mathrm{C}_{2} \mathrm{H}_{2}$ & $2.45 \mathrm{GHz}$ & 160 mbar & 0.4 & $500 \mathrm{ml} / \mathrm{min}$ & $94 \%$ & $>90\left(\mathrm{C}_{2} \mathrm{H}_{2}\right)$ & $641 \mathrm{C}_{2} \mathrm{H}_{2} / \mathrm{kWh}$ & 36 \\
\hline $\mathrm{CH}_{4}$ & $\mathrm{C}_{2} \mathrm{H}_{2}$ & Pulsed $2.45 \mathrm{GHz}$ & $15-65 \mathrm{mbar}$ & $2(0.07)$ & $150 \mathrm{ml} / \mathrm{min}$ & $94 \%$ & $71\left(\mathrm{C}_{2} \mathrm{H}_{2}\right)$ & $851 \mathrm{C}_{2} \mathrm{H}_{2} / \mathrm{kWh}$ & 37 \\
\hline \multicolumn{10}{|c|}{ WASTE GAS TREATMENT } \\
\hline $\mathrm{SF}_{6}+\mathrm{N}_{2}+\mathrm{H}_{2} \mathrm{O}$ & & $2.45 \mathrm{GHz}$ & 1 bar & $6(5.4)$ & $\begin{array}{c}80 \mathrm{l} / \mathrm{min} \mathrm{N}_{2}+ \\
12 \mathrm{l} / \mathrm{min}\left(\mathrm{H}_{2} \mathrm{O}+\mathrm{SF}_{6}\right)\end{array}$ & $\begin{array}{c}99.9 \% \\
\text { decomposition }\end{array}$ & & $1 \mathrm{~m}^{3} / \mathrm{kWh}$ & 38 \\
\hline $\mathrm{CFC} 12+\mathrm{H}_{2} \mathrm{O}_{\mathrm{g}}$ & - & $2.45 \mathrm{GHz}$ & 1 bar & 2 & $2 \mathrm{~kg} / \mathrm{h} \mathrm{CFC} 12$ & $\begin{array}{c}99.99 \% \\
\text { decomposition } \\
\end{array}$ & - & $0.5 \mathrm{~kg} / \mathrm{kWh}$ & 39 \\
\hline
\end{tabular}




\begin{tabular}{|c|c|c|c|c|c|c|c|c|c|}
\hline $\operatorname{VOC}\left(\mathrm{CCl}_{4}\right)+\mathrm{Ar}+\mathrm{He}$ & - & $2.45 \mathrm{GHz}$ & 1 bar & 1 & $11 / \mathrm{min} \mathrm{Ar}+5-12000 \mathrm{ppm} \mathrm{CCl}_{4}$ & $\begin{array}{c}\text { Reduction to ppb } \\
\text { level }\end{array}$ & - & $3 \mathrm{~kg} / \mathrm{kWh}$ & 40 \\
\hline \multicolumn{10}{|c|}{ PYROLYSIS/GASIFICATION } \\
\hline $\begin{array}{c}\text { Brown Coal } \\
\text { (Gasification) }+\mathrm{H}_{2} \mathrm{O}_{\mathrm{g}}+ \\
\text { air }+\mathrm{O}_{2}\end{array}$ & Syngas & $915 \mathrm{MHz}(2)$ & 1 bar & 75 each (70) & $\begin{array}{c}90 \mathrm{~kg} / \mathrm{h} \mathrm{coal}+60 \mathrm{~kg} / \mathrm{h} \mathrm{H} \mathrm{H}_{2} \mathrm{O}_{\mathrm{g}} \\
320 \mathrm{l} / \mathrm{min} \mathrm{N_{2 }}+410 \mathrm{l} / \mathrm{min} \mathrm{\textrm {O } _ { 2 }}\end{array}$ & $99.8 \% \mathrm{CCE}$ & $40\left(\mathrm{H}_{2}\right) \& 32(\mathrm{CO})$ & $84 \%$ CGE & 22 \\
\hline $\begin{array}{l}\text { Charcoal (Gasification) } \\
\quad+\mathrm{H}_{2} \mathrm{O}_{\mathrm{g}}+\text { air }\end{array}$ & Syngas & $2.45 \mathrm{GHz}$ & 1 bar & $6(5)$ & $\begin{array}{c}1.26 \mathrm{~kg} / \mathrm{h} \text { charcoal }+ \\
1.1 \mathrm{~kg} / \mathrm{h} \mathrm{H} \mathrm{O}_{\mathrm{g}}+20 \mathrm{l} / \mathrm{min} \text { air }\end{array}$ & $52 \% \mathrm{CCE}$ & - & $43 \% \mathrm{CGE}$ & 41 \\
\hline $\begin{array}{l}\text { Wood (Pyrolysis) }+ \\
\qquad \mathrm{N}_{2}+\mathrm{Ar}\end{array}$ & Syngas & $\begin{array}{l}\text { Plasma jet } \\
2.45 \mathrm{GHz}\end{array}$ & 200 mbar & $2(1)$ & $\begin{array}{c}11 / \mathrm{min} \mathrm{N}_{2}+11 / \mathrm{min} \text { Ar plasma } \\
10 \mathrm{~g} \text { samples }\end{array}$ & $21 /$ min syngas & - & - & 42 \\
\hline $\begin{array}{c}\text { Waste paper } \\
\text { (Gasification) + air }\end{array}$ & Fuel gas & $2.45 \mathrm{GHz}$ & $1 \mathrm{bar}$ & 0.8 & $1-41 /$ min air +5 g sample & $3.3 \mathrm{~m}^{3} / \mathrm{kg}$ paper & $7.7\left(\mathrm{H}_{2}\right) \& 8.5(\mathrm{CO})$ & $2.3 \mathrm{MJ} / \mathrm{m}^{3} \mathrm{LHV}$ & 43 \\
\hline $\begin{array}{c}\text { Rice straw (Pyrolysis) + } \\
\mathrm{N}_{2} \\
\end{array}$ & Syngas & $2.45 \mathrm{GHz}$ & 1 bar & $2(0.5)$ & $5 \mathrm{~g} \mathrm{sample}+50 \mathrm{ml} / \mathrm{min} \mathrm{N}_{2}$ & $\begin{array}{l}80 \% \text { mass } \\
\text { reduction ratio }\end{array}$ & $\begin{array}{l}55\left(\mathrm{H}_{2}\right) ; 17\left(\mathrm{CO}_{2}\right) \\
13(\mathrm{CO}) ; 10\left(\mathrm{CH}_{4}\right) \\
\end{array}$ & - & 44 \\
\hline \multicolumn{10}{|c|}{$\mathrm{CO}_{2}$ UTILIZATION } \\
\hline $\mathrm{CO}_{2}$ and $\mathrm{H}_{2} \mathrm{O}_{\mathrm{g}}$ & Syngas & Pulsed $915 \mathrm{MHz}$ & $40 \mathrm{mbar}$ & 1 & 3-6 $\mathrm{l} / \mathrm{min} \mathrm{CO}_{2}$ and $\mathrm{H}_{2} \mathrm{O}_{\mathrm{g}}$ & $\begin{array}{c}0.72 \mathrm{~mol} / \mathrm{h} \mathrm{CO} \\
0.22 \mathrm{~mol} / \mathrm{h} \mathrm{H}_{2}\end{array}$ & - & $\begin{array}{c}0.02 \mathrm{~kg} \\
\text { syngas } / \mathrm{kWh}\end{array}$ & 45 \\
\hline $\mathrm{CO}_{2}$ & $\mathrm{CO}$ & $2.45 \mathrm{GHz}$ & $10-250$ mbar & $1(0.6)$ & $51 / \mathrm{min}$ & $30 \%$ & - & $0.17 \mathrm{~kg} \mathrm{CO} / \mathrm{kWh}$ & 46 \\
\hline $\mathrm{CO}_{2}$ and $\mathrm{H}_{2}$ & Syngas & $2.45 \mathrm{GHz}$ & 20-50 mbar & 0.2 & $400 \mathrm{ml} / \mathrm{min} \mathrm{CO}_{2}+\mathrm{H}_{2}$ & $85 \%$ & $100\left(\mathrm{CO}_{2} \rightarrow \mathrm{CO}\right)$ & $0.03 \mathrm{~kg} \mathrm{CO} / \mathrm{kWh}$ & 47 \\
\hline \multicolumn{10}{|c|}{$\mathrm{H}_{2}$ PRODUCTION } \\
\hline $\mathrm{CH}_{4}$ & $\mathrm{H}_{2}$ & $2.45 \mathrm{GHz}$ & 1 bar & $6(1-5)$ & $87.5 \mathrm{l} / \mathrm{min} \mathrm{CH}_{4}$ & $\begin{array}{c}99.8 \%(0.87 \mathrm{~kg} \\
\left.\mathrm{H}_{2} / \mathrm{h}\right)\end{array}$ & $100\left(\mathrm{H}_{2}\right)$ & $640 \mathrm{~g} \mathrm{H}_{2} / \mathrm{kWh}$ & 24 \\
\hline $\mathrm{C}_{2} \mathrm{H}_{2} \mathrm{~F}_{4}$ & $\mathrm{H}_{2}$ & $2.45 \mathrm{GHz}$ & 1 bar & $6(1-5)$ & $97 \mathrm{l} / \mathrm{min} \mathrm{C}_{2} \mathrm{H}_{2} \mathrm{~F}_{4}$ & $84 \%\left(0.7 \mathrm{~kg} \mathrm{H}_{2} / \mathrm{h}\right)$ & $99.9\left(\mathrm{H}_{2}\right)$ & $670 \mathrm{~g} \mathrm{H}_{2} / \mathrm{kWh}$ & 24 \\
\hline $\mathrm{CO}+\mathrm{H}_{2} \mathrm{O}_{\mathrm{g}}$ & $\mathrm{H}_{2}$ & $\begin{array}{l}\text { Reverse Vortex } \\
2.45 \mathrm{GHz}\end{array}$ & 1 bar & 3 & $27 \mathrm{l} / \mathrm{min} \mathrm{H}_{2} \mathrm{O}_{\mathrm{g}}+3 \mathrm{l} / \mathrm{min} \mathrm{CO}$ & $85 \%(\mathrm{CO})$ & $36\left(\mathrm{H}_{2}\right)$ & $3.40 \mathrm{~g} \mathrm{H}_{2} / \mathrm{kWh}$ & 48 \\
\hline $\begin{array}{c}\mathrm{C}_{2} \mathrm{H}_{5} \mathrm{OH} / \mathrm{CH}_{3} \mathrm{OH}+\mathrm{Ar}+ \\
\mathrm{H}_{2} \mathrm{O}_{\mathrm{g}} \\
\end{array}$ & $\mathrm{H}_{2}$ & $2.45 \mathrm{GHz}$ & 1 bar & $0.2-0.7$ & $\begin{array}{c}2 \mathrm{l} / \mathrm{min} \mathrm{Ar}+\mathrm{CH}_{3} \mathrm{OH}+\mathrm{H}_{2} \mathrm{O}_{\mathrm{g}} \\
0.5 \mathrm{l} / \mathrm{min} \mathrm{Ar}+\mathrm{C}_{2} \mathrm{H}_{5} \mathrm{OH}+\mathrm{H}_{2} \mathrm{O}_{\mathrm{g}}\end{array}$ & $100 \%\left(\mathrm{CH}_{3} \mathrm{OH}\right)$ & - & $0.41 \mathrm{~g} \mathrm{H}_{2} / \mathrm{kWh}$ & 49 \\
\hline $\mathrm{CH}_{3} \mathrm{OH}+\mathrm{N}_{2}$ & $\mathrm{H}_{2}$ & $2.45 \mathrm{GHz}$ & 1 bar & $5(1.4)$ & $12.4 \mathrm{l} / \mathrm{min} \mathrm{N}_{2}\left(5 \% \mathrm{CH}_{3} \mathrm{OH}\right)$ & $99 \%$ & $85\left(\mathrm{H}_{2}\right)$ & $2 \mathrm{~g} \mathrm{H}_{2} / \mathrm{kWh}$ & 50 \\
\hline $\begin{array}{l}\text { PFO (pyrolysis fuel oil) } \\
+ \text { Ar }\end{array}$ & $\mathrm{H}_{2}$ & $2.45 \mathrm{GHz}$ & $1 \mathrm{bar}$ & $2(0.85)$ & 5 1/min Ar & $\begin{array}{l}446.3 \mathrm{ml} / \mathrm{min}_{2} \\
27.4 \mathrm{ml} / \mathrm{min} \mathrm{C}_{4}\end{array}$ & $92\left(\mathrm{H}_{2}\right) \& 5\left(\mathrm{C}_{4}\right)$ & $2.8 \mathrm{~g} \mathrm{H}_{2} / \mathrm{kWh}$ & 51 \\
\hline $\begin{array}{c}\mathrm{CH}_{3} \mathrm{OH} / \mathrm{C}_{2} \mathrm{H}_{5} \mathrm{OH} / \\
\mathrm{C}_{3} \mathrm{H}_{7} \mathrm{OH}+\mathrm{Ar}+\mathrm{H}_{2} \mathrm{O}_{\mathrm{g}}\end{array}$ & $\mathrm{H}_{2}$ & $\begin{array}{l}\text { Tornado-type } \\
2.45 \mathrm{GHz}\end{array}$ & $1 \mathrm{bar}$ & $2(0.45)$ & $\begin{array}{c}11 / \mathrm{min} \mathrm{Ar}+9 \mathrm{ml} / \mathrm{min}\left(\mathrm{CH}_{3} \mathrm{OH}\right) \\
1 \mathrm{l} / \mathrm{min} \mathrm{Ar}+13.5 \mathrm{ml} / \mathrm{min}\left(\mathrm{C}_{2} \mathrm{H}_{5} \mathrm{OH}\right) \\
11 / \mathrm{min} \mathrm{Ar}+8.5 \mathrm{ml} / \mathrm{min}\left(\mathrm{C}_{3} \mathrm{H}_{7} \mathrm{OH}\right)\end{array}$ & $\begin{array}{l}0.10 \mathrm{~g} \mathrm{H}_{2} / \mathrm{h} \\
0.24 \mathrm{~g} \mathrm{H}_{2} / \mathrm{h} \\
0.18 \mathrm{~g} \mathrm{H}_{2} / \mathrm{h}\end{array}$ & $>95\left(\mathrm{H}_{2}\right)$ & $\begin{array}{l}0.24 \mathrm{~g} \mathrm{H}_{2} / \mathrm{kWh} \\
0.53 \mathrm{~g} \mathrm{H}_{2} / \mathrm{kWh} \\
0.45 \mathrm{~g} \mathrm{H}_{2} / \mathrm{kWh}\end{array}$ & 52 \\
\hline
\end{tabular}




\subsection{Capital cost}

The capital cost of conventional chemical reactors is mainly determined by the reactor dimensions (volume) and material of construction. In the case of MWP reactors, the main driver of the total capital investment is the microwave generator in combination with the waveguide components required to operate the plasma reactor. Figure 2 gives an estimation of the commercial price of MWP equipment (generator + components, see Table 1) as well as the commercial equipment cost per $\mathrm{kW}$ microwave power, as function of the total output power of the microwave generator. For the particular case of 100 $\mathrm{kW}$, the equipment cost amounts to $\sim 1400 € / \mathrm{kW}$ microwave power. This value is calculated as the ratio between the MWP equipment price and the total output power of the generator (e.g. $1.410^{5} € / 100$ $\mathrm{kW}$ ). The prices given in Figure 2 include switch mode HV power supplies, which are required in the case of atmospheric pressure plasmas; the switch mode has low ripple and better control on the magnetron including the rise time or time needed for the magnetron to increase the power from $0 \mathrm{~W}$ to the requested power for plasma ignition. It is observed that the higher the output power of the microwave generator, the lower the equipment cost per $\mathrm{kW}$ microwave power as shown in Figure 2 (black line, square markers). Therefore, the development of single unit microwave sources with higher power (> $100 \mathrm{~kW}$ ) should significantly reduce the high capital cost of MWP equipment.

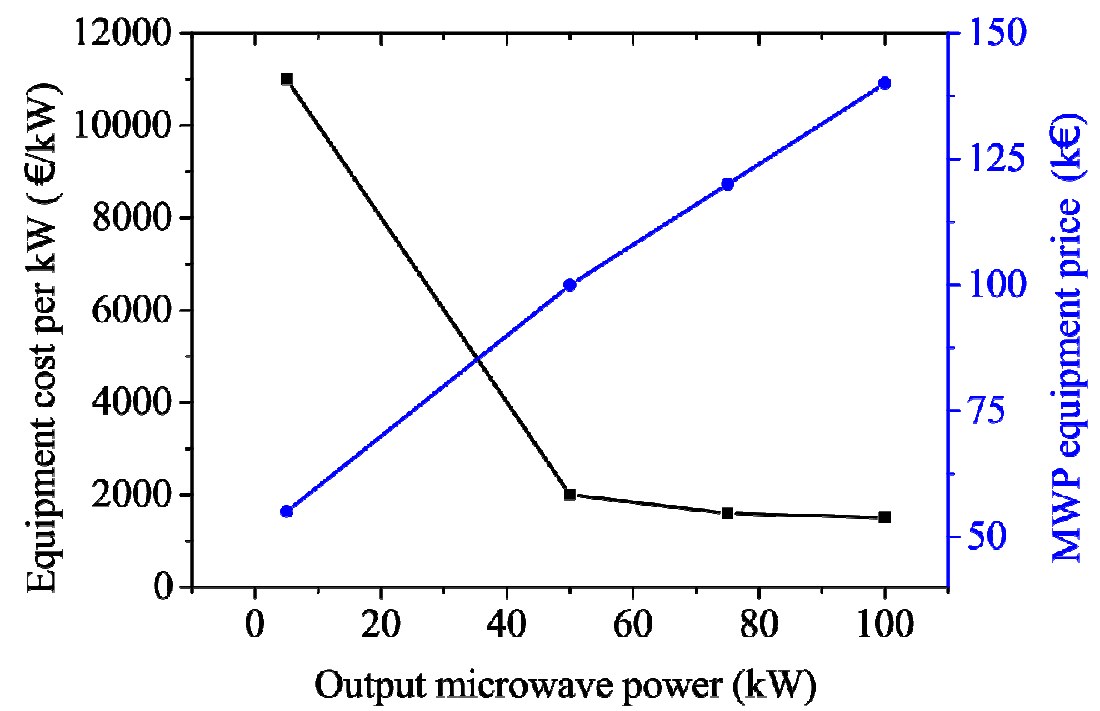

Output microwave power $(\mathrm{kW})$

20 Figure 2. Commercial MWP equipment price (generator + components, see Table 1) and commercial equipment cost per $\mathrm{kW}$ microwave power (MWP equipment price $(\mathrm{k} €$ ) divided by output microwave power $(\mathrm{kW})$ ) as function of the total output power of the microwave generator for equipment at 915 $\mathrm{MHz}$ frequency (microwave generator based on a switch-mode HV power supply).

\subsection{Industrial applications of MWP technology}

The applications of MWP at industrial-scale have been previously discussed in the literature. ${ }^{16}$ In summary, the main industrial applications include: photoresist stripping in semiconductor manufacturing, deposition of barrier layers in PET bottles, high rate deposition process of quarts on 
1 polycarbonate windows, plasma photo curing of paintings applied to the automotive industry, UV

2 disinfection for water treatment, waste gas treatment for decomposition of fluorine-based components

3 such as $\mathrm{CF}_{4}, \mathrm{C}_{2} \mathrm{~F}_{6}, \mathrm{CHF}_{3}$, and $\mathrm{SF}_{6}{ }^{38,53}$ or ammonia, and plasma reforming to increase efficiency in

4 wood gas engines. In the past few years, few advancements have been reported on the industrial

5 application of this technology, although there are some novel processes that should be added to the existing list. Most of the future industrial applications of the MWP technology will be relevant to high temperature processes for chemical synthesis and (oxygenated) fuels conversion including pyrolysis, gasification and reforming of organic waste, biomass, and fossil fuels. Other application fields in which MWP will play a role are water and air purification, material synthesis (nano-particle production, diamonds, textiles) and biomedicine (cancer treatment, wound healing, disinfection).

Table 3 presents these applications together with a brief description of the main features of the process. Most of the future industrial applications of the MWP technology will be relevant to high temperature processes for chemical synthesis and (oxygenated) fuels conversion including pyrolysis, gasification and reforming of organic waste, biomass, and fossil fuels. Other application fields in which MWP will play a role are water and air purification, material synthesis (nano-particle production, diamonds, textiles) and biomedicine (cancer treatment, wound healing, disinfection).

Table 3. Summary of novel MWP processes for chemical applications.

\begin{tabular}{|c|c|c|}
\hline PROCESS/(COMPANY) & DESCRIPTION & MAIN FEATURES \\
\hline $\begin{array}{l}\text { BIOMASS } \\
\text { GASIFICATION } \\
\text { (Plasma2Energy) }^{23}\end{array}$ & $\begin{array}{l}\text { - Medium-scale plant for biomass } \\
\text { gasification. } \\
\text { - It runs for four years. } \\
\text { - It exploited the concept of coupling } \\
\text { multiple microwave generators to a } \\
\text { single gasification chamber. } \\
\text { - The plant consumed only } 20 \% \text { of } \\
\text { the energy generated. }\end{array}$ & $\begin{array}{l}\text { - Input microwave power }=30 \mathrm{~kW} \text { (plasmatrons) } \\
\text { - Pressure (bar) }=1 \\
\text { - Annual Biomass Capacity = } 3.3 \mathrm{kton} \\
\text { - Selectivity }\left(\mathrm{H}_{2}\right)=50-52 \% \\
\text { - Annual Production }=1830 \mathrm{~m}^{3} \text { ethanol \& } 253 \mathrm{~m}^{3} \\
\text { diesel fuel } \\
\text { - Maintenance: } 5 \text { years } \\
\text { - Lifetime: } 25 \text { years }\end{array}$ \\
\hline $\begin{array}{l}\text { CARBON FIBER } \\
\text { MANUFACTURING } \\
\text { (RMX Technologies) }^{54}\end{array}$ & $\begin{array}{l}\text { - Low-pressure microwave plasma } \\
\text { enhanced the oxidation and } \\
\text { carbonization steps. } \\
\text { - Reduction in the residence time and } \\
\text { the equipment size by } 1 / 3 \text {. } \\
\text { - Energy requirements were reduced } \\
\text { by } 75 \% \text { and manufacturing costs by } \\
20 \% \text { compared to conventional one. }\end{array}$ & $\begin{array}{l}\text { - Input MW power }=30 \mathrm{~kW}(915 \mathrm{MHz}) \\
\text { - Pressure }(\mathrm{mbar})=10 \\
\text { - } \text { Reactor size: } \text { Diameter }=0.05 \mathrm{~m} \& \text { Length }=4 \mathrm{~m} \\
\text { - Energy efficiency }=17 \mathrm{kWh} / \mathrm{kg} \text { carbon fiber }\end{array}$ \\
\hline $\begin{array}{l}\text { PECVD OF } \mathrm{Si}_{3} \mathrm{~N}_{4} \mathrm{ON} \\
\text { MULTI-CRYSTALLINE } \\
\text { SILICON } \\
\text { SOLAR CELLS }\end{array}$ & $\begin{array}{l}\text { - Deposition of silicon nitride anti- } \\
\text { reflective layers on solar cell } \\
\text { wafers by plasma enhanced carbon } \\
\text { vapour deposition (PECVD). }\end{array}$ & $\begin{array}{l}\text { - Input microwave power }=2 \times 4 \mathrm{~kW} \text { (pulsed-type) } \\
\text { - Pressure }(\mathrm{mbar})=0.01-1 \\
\text { - Reactor size: Diameter }=0.02 \mathrm{~m} \& \text { Length }=1.5 \mathrm{~m} \\
\text { - Production }=1500 \text { solar cells wafers per hour }\end{array}$ \\
\hline
\end{tabular}




\begin{tabular}{|c|c|c|}
\hline $\begin{array}{c}\text { TREATMENT } \\
\text { OF CHRONIC WOUNDS } \\
\text { (Adtec Europe SteriPlas) }^{56}\end{array}$ & $\begin{array}{l}\text { - Wound healing by reduction of } \\
\text { microbial load and by modifying } \\
\text { the wound microenvironment. } \\
\text { - Working gas is Argon, which } \\
\text { ensures the reproducibility of } \\
\text { generated active agents. }\end{array}$ & $\begin{array}{l}\text { - Input microwave power }=200 \mathrm{~W} \\
\text { - Working gas }=\text { Argon, purity } 99.95 \% \\
\text { - Operating temp range }=10-30{ }^{\circ} \mathrm{C}\end{array}$ \\
\hline $\begin{array}{l}\text { PRODUCTION OF } \\
\text { SYNTHETIC DIAMOND } \\
\text { MPECVD }(\text { ASTeX })^{57}\end{array}$ & $\begin{array}{l}\text { - Synthetic diamond growth from the } \\
\text { gas phase by microwave-plasma } \\
\text { enhanced vapor chemical } \\
\text { deposition (MPECVD). } \\
\text { - Synthesis diamonds are presented } \\
\text { as a much affordable option over } \\
\text { naturally mined diamonds. }\end{array}$ & $\begin{array}{l}\text { - Frequency }=915 \mathrm{MHz} \& \text { Power }=90 \mathrm{~kW} \\
\text { - Pressure (torr) }=180 \& \text { Gas temperature }=4000 \mathrm{~K} \\
\text { - } \text { Working gas }=\mathrm{H}_{2}+1-5 \% \mathrm{CH}_{4} \\
\text { - Deposition rate }=1 \mathrm{~g} / \mathrm{h} \\
\text { - Annual production rate }=214300 \text { carats (10 reactors) } \\
\text { - Diamond production cost }=14 \$ \text { carat }\end{array}$ \\
\hline
\end{tabular}

\section{State of development and outlook}

\subsection{Current status of the technology}

In this section, the technical and operational limitations of the technology are discussed. The different possibilities to scaling up MWP technology are also addressed. Finally, the most relevant scientific and engineering challenges as for the implementation of this technology to commercial scale are presented in the form of a timeline.

\subsubsection{Microwave generator}

The largest single-unit continuous wave $(\mathrm{CW})$ microwave generator, so-called magnetron, presents a limitation of maximum output power of $15 \mathrm{~kW}$ at a frequency of $2450 \mathrm{MHz}$ and $100 \mathrm{~kW}$ at $915 \mathrm{MHz}$. There are magnetrons, operating at $15 \mathrm{~kW}$ max power, which are used for diamond synthesis at very low pressure; however, their performance seems to be quite poor and their lifetime rather short (few thousand hours range). In contrast, $15 \mathrm{~kW}$ generators work relatively well for laboratory applications, as those do not involve $24 \mathrm{~h}$ operation. From an economic and regulatory point-of-view, there are two commercially preferable frequencies on the ISM bands, $915 \mathrm{MHz}$ (L-band) and $2450 \mathrm{MHz}$ (S-band) ${ }^{54}$ that can be used for MWP reactors. The emissions at other frequencies can create electromagnetic interference and disrupt radio communications. To date, most of the work with MWP has been done at the standard microwave frequency of $2450 \mathrm{MHz}$. Microwave generators at this frequency, which have been largely produced for decades (e.g. domestic microwave ovens), offer various benefits such as relatively high power capability, durability, low cost and compact size of MWP elements. In the case of the $915 \mathrm{MHz}$ frequency, the waveguide components are characterized by larger sizes (about three times larger than those of one at $2450 \mathrm{MHz}$ ), which makes these microwave generators more costly compared to $2450 \mathrm{MHz}$ generators. There are also devices operating at non-standard frequencies (20$250 \mathrm{GHz}$ ), known as travelling wave tubes or gyrotrons, with maximum output powers of 1-2 MW. These high power sources are mostly used in nuclear fusion and their evaluation is out of the scope of this paper. 


\subsubsection{Plasma ignition, stability and uniformity}

Plasma is ignited when the applied electric field strength overcomes the breakdown voltage of the working gas, which is called electric breakdown. Data for the breakdown voltage for different atomic and molecular gases as a function of the pressure and distance between two parallel electrodes, termed the Paschen curves, are well documented in the literature. ${ }^{11}$ When low pressure MWP is considered, the required field strength to ignite and maintain the plasma is less demanding compared to MWP at atmospheric pressure where a rather high electron collision frequency is needed to energize the plasma. For large scale chemical applications, uniformity and stability become imperative in the production process, as it is highly important to ensure a constant and reproducible product composition. One of the major challenges for use of the MWP technology is its inherent instability, which has implications in reproducibility of the results. When operating MWP at atmospheric pressure and high power conditions, plasma stability depends mostly on the interplay between input microwave power and flow dynamics (working gas flow rate, feed gas composition, swirl flow). To obtain stable plasmas, it is important that the input power is adapted to the flow conditions. If the input power is excessive in relation to the gas flow, it can cause arcing and overheating of the quartz tube, ${ }^{30}$ whereas if the power is too low, the degree of ionization along with gas bulk temperature will decrease resulting in plasma extinction. For high power equipment, a motorized sliding short circuit and automatic tuner can be used to compensate for variations of the reflected power, which represents a measure of plasma stability. However, operating at a minimum reflected power implies operation very close to or at unstable conditions that can lead to plasma loss or fluctuations (non-uniform). ${ }^{58,59}$ Another reason of instability is small fluctuations in the microwave frequency, which causes variations in the electron density, largely influencing the plasma frequency. ${ }^{60}$ In this regard, there are a number of practical measures to improve plasma stability: 1) addition of a carrier/working gas; argon, helium, nitrogen, air and water are the most commonly used gases, 2) design of a novel reactor configuration such as "Vortex/Tornado-type"48, 61 or multi-point microwave coupling ${ }^{62}$, 3) combination of microwave and other fields (e.g. radio-frequency) $)^{15}$ and 4) insulation of the reactor, which plays an important role in enhancing the stability by reducing heat losses.

\subsubsection{Cooling of the plasma reactor}

MWP is characterized by high power densities, which enable MWP reactors to achieve energy efficiencies up to $90 \%$. As a result, one of the major technical challenges is the cooling of the reactor due to the high values of power input per unit wall area $\left(\mathrm{W} / \mathrm{cm}^{2}\right)$, which increases significantly the chance of reactor melting (quartz tube). Hence, MWP reactors require carefully designed cooling systems to ensure a continuous operation. The most common cooling techniques are forced-air/ $\mathrm{N}_{2}$ in combination with cooling water jacket-type ${ }^{47}$ or, in more demanding cases, cooling oil, surrounding the plasma reactor. The area with the highest risk for reactor puncturing is the one found within the 
1 ignition plasma zone, i.e. where the waveguide crosses the quartz tube, which also presents the highest 2 electric field strength. Frequently, MWP reactors are characterized by a one-sided incoming microwave field, meaning that the plasma is mostly energized on one side. This causes nonhomogeneous plasma formation, which leads to hot spot formation. ${ }^{62}$ When the power input per unit wall area becomes relatively high $\left(>40 \mathrm{~W} / \mathrm{cm}^{2}\right),{ }^{14}$ a common measure of protection of the reactor wall is the use of high-speed tangential gas injection (swirl flow) to confine the plasma at the core of the reactor by creating a tornado/vortex gas motion, which isolates the reactor wall from the plasma column. Another alternative is the addition of an inert gas such as helium with high heat conductivity to increase the dissipation of heat generated in the plasma, thus reducing the possibility of reactor failure. Therefore, thermal management is one of the key engineering challenges to continuously operate this type of systems at large scale. Note that MWP reactors can be run under two different conditions: a) non-thermal/low pressure MWP and b) thermal/atmospheric pressure MWP. In the latter, the cooling requirements are rather demanding, as the temperature of the gas gets close to the temperature of the electrons - all species are close to thermal equilibrium - whereas in non-thermal MWP, the bulk gas remains rather cold (near room temperature) while having high kinetic electron temperature.

\subsubsection{Reactor material}

As already stated, one of the main features provided by MWP is the high energy density, which also implies high temperatures inside the reactor. In this regard, the material of the reactor is a crucial aspect in MWP operation and must fulfill three requirements: 1) have high melting point to provide resistance to high temperature operation, 2) have thermal shock resistance and 3) be transparent to microwaves, i.e. it should not absorb or reflect microwaves. At lab-scale, the most commonly used material is quartz, which seems unsuitable for commercial applications due to its fragility. Therefore, other materials such as ceramics (alumina-based), aluminium oxynitride (melting point above 2000 ${ }^{\circ} \mathrm{C}$ ) or silicon carbide can be used to build large size MWP reactors. The latter has already been used in a plasma gasification unit. ${ }^{63}$ Moreover, in the pilot-scale setup developed by Uhm et al. $2014,{ }^{22}$ alternative materials such as HACT180 (fire-resistance ceramic) and INCT120 (insulating-cement) were used to form the inner and outer layers of the MWP reactor respectively, showing great performance at temperatures up to $1800{ }^{\circ} \mathrm{C}$.

\subsubsection{Process control and safety}

MWP-based processes show remarkably fast dynamics, in which most of the events take place in the micro/milli-second range. Such dynamics require demanding continuous process control tools that are capable of adjusting process parameters within a response time of milliseconds. Various control parameters are critical during MWP operation: 1) net input microwave power, 2) input gas flow rate, 3) operating pressure and 4) cooling flow rate. 
1 The input power is the most important process variable, as it influences directly the absorbed energy

2 by the plasma and consequently the temperature of the reactor. A practical way to control the forward power, while assuring reactor stability is by measuring the reactor wall temperature. This value is then compared to a setpoint that guarantees no reactor puncturing. The input power is then regulated based on the temperature difference between the readings and the setpoint. The gas flow rate largely affects reactor stability as low flow rates can lead to severe increase in the specific energy input (SEI, $\mathrm{J} / \mathrm{m}^{3}$ ), i.e. the ratio between the input power and the inlet flow rate, causing rupture of the reactor. When the flow rates are excessive, plasma is extinguished due to the drop of SEI. The study of operating flow rate range should therefore be carefully assessed in MWP reactors. Control of the operating pressure is particularly important when working with low-pressure MWP, as it influences both plasma ignition and sustenance. When pressure is increased, the number of collisions between electrons and other species also increases, implying that more input power is needed to sustain plasma.

Finally, as discussed, the cooling system (flow rate) requires a suitable design in which sufficient heat is removed from the plasma reactor to ensure proper function and stability of the system. With respect to safety, the primary concern is related to exposure of operators and/or fuel to microwave radiation. Thus, the installation of microwave leak detectors is highly recommended when operating MWP equipment. In this regard, it is critical to assure good microwave shielding to eliminate any possibility of microwave radiation. If openings along the cavity are present, common practice is the installation of a metal mesh as an additional shield. Furthermore, considering the possible risk of reactor breaking, it is advisable to operate MWP reactors within a properly ventilated area to contain the hazard of a gas leakage.

\subsection{Scalability}

When evaluating the development of a new process, the production capacity represents the main design guideline, thus dictating the equipment requirements. Bulk chemicals are commonly produced on a very large scale, implying the need to operate at considerably high throughput and therefore demanding high energy input. As an example, a common methanol manufacturing plant ${ }^{64}$ with a production rate of $100 \mathrm{kton} / \mathrm{year}$ and electricity usage of $550 \mathrm{kWh} / \mathrm{ton}$ is herein considered. The largest MWP unit developed so far was able to handle $3.3 \mathrm{kton}$ biomass/year resulting in a production of 1.5 kton ethanol/year. ${ }^{23}$ Hence, to meet the expected capacity for bulk chemical manufacturing, the current MWP reactors should be scaled up by a factor of 66 for this particular process, which is challenging. Another pilot-scale MWP gasification unit was developed by Uhm et al. (2014). ${ }^{22}$ In their work, two microwave generators of $75 \mathrm{~kW}$ output each were attached to the gasification chamber and enabled inlet flow rates of 2.2 ton coal/day with respective throughput of 1.9 ton syngas/day corresponding to a total calorific value of $0.5 \mathrm{MW}_{\text {th }}$. To date, the use of MWP reactors in bulk chemical manufacturing processes has been hampered mostly because the input microwave power required to sustain the 
1 plasma at rather high throughput cannot be directly delivered by the existing microwave generators.

2 To our knowledge, there are two possibilities to address this technical limitation: 1) coupling multiple

3 microwave generators to a single reactor chamber or 2) developing single unit microwave sources with

$4>100 \mathrm{~kW}$ output power at lower frequencies (e.g. $433 \mathrm{MHz}$ ).

5 The first approach has already been explored for a medium-scale gasification plant as shown in

6 Sanchez A.L. (2010). ${ }^{23}$ To increase the capacity, multiple $20 \mathrm{~kW}$ microwave plasmatrons were arranged around and along a single MWP gasification chamber. In a plasmatron, plasma is formed as an axial, cylindrical extension of the inner conductor of a coaxial line; microwave power is fed from a standard waveguide and a waveguide-to-coaxial transition carries out the wave mode conversion and impedance matching functions. A general advantage of these plasma sources is homogeneous plasma heating from all sides. ${ }^{62,}{ }^{65} \mathrm{~A}$ schematic representation is presented in Figure 3. When multiple microwave sources are to be combined, one should take into account the minimization of crosscoupling between generators, as this can cause a drastic reduction of the generator life and also nonuniformity of the energy delivered to the plasma. A more detailed description regarding design and application of multiple microwave generators is discussed in references ${ }^{14,62,66}$. As a final remark, the MWP gasification system reported in Sanchez A.L. (2010) ${ }^{23}$ was designed to have maintenance every 5 years and a lifetime of 25 years, which is common practice in the chemical process industry. The second alternative envisages the development of single unit microwave sources with $>100 \mathrm{~kW}$ output power. Within the microwave ISM frequencies for industrial processing, the frequency band $433.05-434.79 \mathrm{MHz}$ (central frequency $433.92 \mathrm{MHz}$ ) appears to be the most interesting one for scale-up. Currently, there are no reported industrial applications operating at $433.96 \mathrm{MHz}$. However, according to magnetron manufacturers, $\mathrm{CW}$ magnetrons operated at this frequency can be designed to deliver much higher microwave power levels than the L-band $(896 \mathrm{MHz}, 915 \mathrm{MHz}, 922 \mathrm{MHz}$ and 929 $\mathrm{MHz}$ ) magnetrons namely, between 0.5 and $1 \mathrm{MW}$. The design of high power $433.96 \mathrm{MHz}$ equipment should consider the development not solely of the magnetron and the HV DC power supply, but also all the high power rated WR2100 waveguide components (isolators, impedance tuners etc.) required to run industrial applications. 


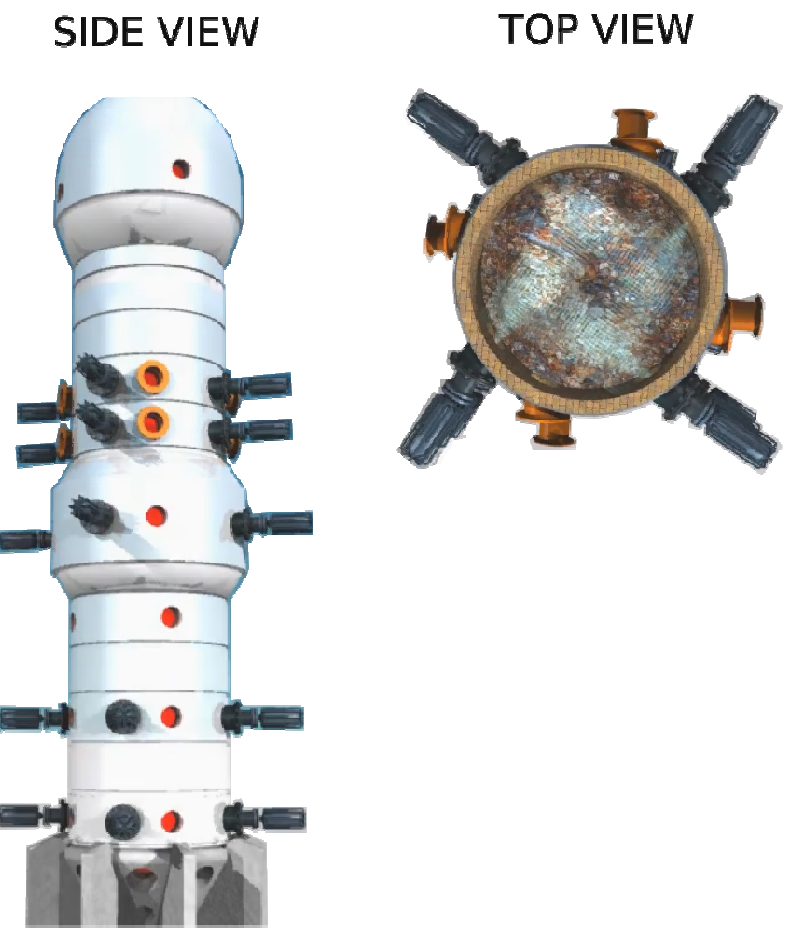

Figure 3. Schematic representation of multiple microwave generators attached to a single reactor. ${ }^{23}$ Note that each of the black elements represents a $20 \mathrm{~kW}$ microwave plasmatron.

\subsection{Potential of MWP for commercial chemical applications}

At the current technological state of MWP, the concept of modularized production seems to be the most promising approach to respond to: (1) the decentralized electricity generation via renewable energy sources, and (2) the present volatile markets due to frequent fluctuations in factors such as demand, economic uncertainties, depletion of natural resources, demographic trends and oil and gas prices among others. ${ }^{67}$ In this regard, the development of modular MWP units powered by locally generated renewable electricity for distributed manufacturing may, at least partially, change the current model of very large scale centralized industrial processing and also form an attractive strategy to overcome rapid changes in the market demand. ${ }^{68}$ Therefore, the production of syngas, hydrogen, acetylene and localized waste treatment represent some of the opportunities that MWP technology can address at present.

As for bulk chemical manufacturing, the first steps to bring MWP technology to commercial scale have been taken, although some technical and operational challenges still need to be tackled before MWP can be extensively used in the chemical industry. Figure 4 presents in the form of a timeline, the main scientific and engineering challenges to be addressed. These challenges mainly concern: 1) development of higher than $100 \mathrm{~kW}$ microwave power sources and of effective plasma reactor designs that can be powered by multiple microwave generators to attain wide throughput range, 2) development of suitable reactor materials for MWP operation, 3) improvement of process reliability 
1 (controllability, stability and uniformity) and 4) development of chemical kinetic models that can be

2 implemented into multidimensional multiphysics models for process design, optimization, and control.

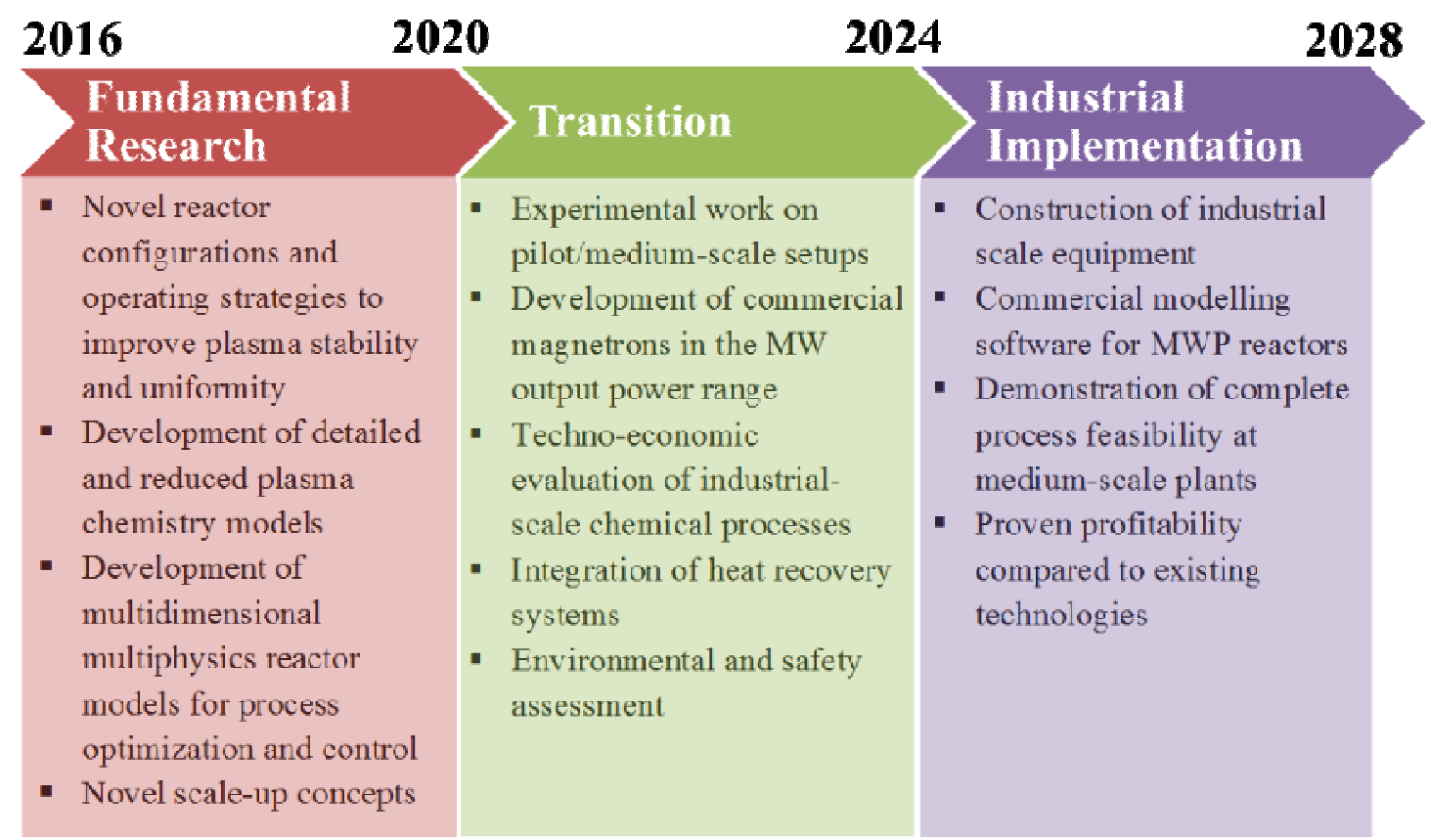

Figure 4. Timeline for the implementation of MWP technology in chemical manufacturing industry.

\section{Conclusions}

Microwave plasma (MWP) is one of the most promising enabling technologies for electricity-based reactors as regards the future partial electrification of the chemical industry. In this work, we have summarized the extensive research carried out on MWP at laboratory-scale combined with some successfully demonstrated industrial applications. Concerning chemical processing applications, high temperature processes, such as pyrolysis, gasification and reforming of organic waste, biomass and fossil fuels have the highest potential to benefit from MWP. However, it is imperative to perform research with medium-scale setups to quantitatively demonstrate the profitability, reliability and operational benefits of the technology, as already shown for biomass gasification. In parallel, work on development of a) single-unit microwave sources with $>100 \mathrm{~kW}$ output power (0.5-1 MW), b) suitable reactor materials that can withstand harsh operating conditions and c) reaction kinetic models that can be implemented into multidimensional multiphysics reactor models appear to be key scientific and engineering challenges that need to be addressed to promote wider application of the technology to large scale operations. 
The research leading to these results has received funding from the European Community's Seventh

Framework Programme under grant agreement no. FP7-NMP-2012-309874.

\section{References}

1. Stankiewicz A and Van Gerven T, The Fundamentals of Process Intensification. Wiley (2015).

2. Van Gerven T and Stankiewicz A, Structure, Energy, Synergy, Time-The Fundamentals of Process Intensification. Ind Eng Chem Res 48: 2465-2474 (2009).

3. Kiss AA, Distillation technology-still young and full of breakthrough opportunities. $J$ Chem Technol Biot 89: 479-498 (2014).

4. Chen JF, The Recent Developments in the HiGee Technology, http://inpact.inptoulouse.fr/GPE-EPIC2009/images/presentation_chen.pdf. (accessed 05-10-2016).

5. Lavric D and Woehl P, Advanced-FlowTM glass reactors for seamless scale-up. Chemistry Today 27: 45-48 (2009).

6. Styring P and Jansen D, Carbon Capture and Utilisation in the green economy. The centre for Low Carbon Futures (2011).

7. Mennicken L, Janz A and Roth S, The German R\&D Program for CO2 UtilizationInnovations for a Green Economy. Environ Sci Pollut R 23: 11386-11392 (2016).

8. Wang MY, Wang Z, Gong XZ and Guo ZC, The intensification technologies to water electrolysis for hydrogen production - A review. Renew Sust Energ Rev 29: 573-588 (2014).

9. Hu B, Guild C and Suib SL, Thermal, electrochemical, and photochemical conversion of CO2 to fuels and value-added products. J Co2 Util 1: 18-27 (2013).

10. Boulos MI, The Role of Transport Phenomena and Modeling in the Development of Thermal Plasma Technology. Plasma Chem Plasma P 36: 3-28 (2016).

11. Fridman A, Plasma Chemistry. Cambridge: Cambridge University Press (2008).

12. Argyle MD and Bartholomew $\mathrm{CH}$, Heterogeneous Catalyst Deactivation and Regeneration: A Review. Catalysts 5: 145-269 (2015).

13. Dincer I and Acar C, Review and evaluation of hydrogen production methods for better sustainability. Int J Hydrogen Energ 40: 11094-11111 (2015).

14. Ferreira CM and Moisan M, Microwave Discharges Fundamentals and Applications. NATO ASI Series (1993).

15. Lebedev YA, Microwave discharges: generation and diagnostics. 25th Summer School and International Symposium on the Physics of Ionized Gases - Spig 2010257 (2010).

16. Kaiser M, Baumgartner KM and Mattheus A, Microwave Plasma Sources -Applications in Industry. Contrib Plasm Phys 52: 629-635 (2012).

17. Schulz A, Buchele P, Ramisch E, Janzen O, Jimenez F, Kamm C, Kopecki J, Leins M, Merli S, Petto H, Mendez FR, Schneider J, Schumacher U, Walker M and Stroth U, Scalable Microwave Plasma Sources From Low to Atmospheric Pressure. Contrib Plasm Phys 52: 607614 (2012).

18. Uhm HS, Hong YC and Shin DH, A microwave plasma torch and its applications. Plasma Sources Sci T 15: S26-S34 (2006).

19. Jankowski KJ and Reszke E, Microwave Induced Plasma Analytical Spectrometry. RSCPublishing (2011).

20. Stefanidis GD, Munoz AN, Sturm GSJ and Stankiewicz A, A helicopter view of microwave application to chemical processes: reactions, separations, and equipment concepts. Rev Chem Eng 30: 233-259 (2014).

21. Sturm GSJ, Munoz AN, Aravind PV and Stefanidis GD, Microwave-Driven Plasma Gasification for Biomass Waste Treatment at Miniature Scale. Ieee T Plasma Sci 44: 670-678 (2016). 
22. Uhm HS, Na YH, Hong YC, Shin DH and Cho CH, Production of hydrogen-rich synthetic gas from low-grade coals by microwave steam-plasmas. Int J Hydrogen Energ 39: 4351-4355 (2014).

23. Sanchez AL, Method and apparatus for plasma gasification of carbonic material by means of microwave radiation. US Patent: 2010/0219062 (2010).

24. Jasinski M, Dors M, Nowakowska H, Nichipor GV and Mizeraczyk J, Production of hydrogen via conversion of hydrocarbons using a microwave plasma. J Phys D Appl Phys 44 (2011).

25. Jasinski M, Czylkowski D, Hrycak B, Dors M and Mizeraczyk J, Atmospheric pressure microwave plasma source for hydrogen production. Int J Hydrogen Energ 38: 11473-11483 (2013).

26. Dincer I and Zamfirescu C, Sustainable Hydrogen Production. Elsevier (2016).

27. Banerjee $\mathrm{S}$, Musa $\mathrm{MN}$ and Jaafar $\mathrm{AB}$, Economic assessment and prospect of hydrogen generated by OTEC as future fuel. Int J Hydrogen Energ (2016).

28. Grabner M, Industrial Coal Gasification Technologies Covering Baseline and High-Ash Coal. Wiley-VCH (2014).

29. Choi DH, Chun SM, Ma SH and Hong YC, Production of hydrogen-rich syngas from methane reforming by steam microwave plasma. J Ind Eng Chem 34: 286-291 (2016).

30. Miotk R, Hrycak B, Czylkowski D, Dors M, Jasinski M and Mizeraczyk J, Liquid fuel reforming using microwave plasma at atmospheric pressure. Plasma Sources Sci T 25 (2016).

31. Nakanishi S and Sekiguchi H, Comparison of reforming behaviors of hexane and isooctane in microwave steam plasma. J Jpn Petrol Inst 48: 22-28 (2005).

32. Fall M, Bowlin R and Shulman H, Microwave enhanced cracking of ethane for ethylene production. AIChE Spring Meeting and Global Congress on Process Safety: 1-12 (2012).

33. Mora M, Garcia MD, Jimenez-Sanchidrian C and Romero-Salguero FJ, Transformation of light paraffins in a microwave-induced plasma-based reactor at reduced pressure. Int $J$ Hydrogen Energ 35: 4111-4122 (2010).

34. Kim TS, Song S, Chun KM and Lee SH, An experimental study of syn-gas production via microwave plasma reforming of methane, iso-octane and gasoline. Energy 35: 2734-2743 (2010).

35. Huang J, Badani MV, Suib SL, Harrison JB and Kablauoi M, Partial Oxidation of Methane to Methanol through Microwave Plasmas - Reactor Design to Control Free-Radical Reactions. $J$ Phys Chem-Us 98: 206-210 (1994).

36. Shen CS, Sun YZ, Sun DK and Yang HS, A study on methane coupling to acetylene under the microwave plasma. Sci China Chem 53: 231-237 (2010).

37. Heintze $\mathbf{M}$ and Magureanu $\mathbf{M}$, Methane conversion into acetylene in a microwave plasma: Optimization of the operating parameters. J Appl Phys 92: 2276-2283 (2002).

38. Radoiu M and Hussain S, Microwave plasma removal of sulphur hexafluoride. $J$ Hazard Mater 164: 39-45 (2009).

39. Masahiro B, Development of CFCs Decomposition System Using Microwave Plasma. Mitsubishi Heavy Industries, Ltd 37: 83-87 (2000).

40. Rubio SJ, Quintero MC, Rodero A and Rodriguez JMF, Assessment of a new carbon tetrachloride destruction system based on a microwave plasma torch operating at atmospheric pressure. J Hazard Mater 148: 419-427 (2007).

41. Yoon SJ and Lee JG, Hydrogen-rich syngas production through coal and charcoal gasification using microwave steam and air plasma torch. Int J Hydrogen Energ 37: 17093-17100 (2012).

42. Lupa CJ, Wylie SR, Shaw A, Al-Shamma'a A, Sweetman AJ and Herbert BMJ, Experimental analysis of biomass pyrolysis using microwave-induced plasma. Fuel Process Technol 97: 7984 (2012).

43. Paris Knongkrapan NT, and Tanongkiat Kiatsiriroat, Thermochemical Conversion of Waste Papers to Fuel Gas in a Microwave Plasma Reactor. Journal of Clean Energy Technologies 1: 80-83 (2013).

44. Huang YF, Kuan WH, Lo SL and Lin CF, Total recovery of resources and energy from rice straw using microwave-induced pyrolysis. Bioresource Technol 99: 8252-8258 (2008). 
45. Chen GX, Silva T, Georgieva V, Godfroid T, Britun N, Snyders R and Delplancke-Ogletree MP, Simultaneous dissociation of $\mathrm{CO} 2$ and $\mathrm{H} 2 \mathrm{O}$ to syngas in a surface-wave microwave discharge. Int J Hydrogen Energ 40: 3789-3796 (2015).

46. van Rooij G, van den Bekerom D, den Harder N, Minea T, Berden G, Bongers W, Engeln R, Graswinckel M, Zoethout E and van de Sanden M, Taming microwave plasma to beat thermodynamics in CO2 dissociation. Faraday Discuss 183: 233-248 (2015).

47. de la Fuente JF, Moreno SH, Stankiewicz AI and Stefanidis GD, Reduction of CO2 with hydrogen in a non-equilibrium microwave plasma reactor. Int J Hydrogen Energ.

48. Ma SH, Choi DH, Chun SM, Yang SS and Hong YC, Hydrogen Production by the Water-Gas Shift Reaction Using an Atmospheric Steam Plasma Torch System with a Reverse Vortex Reactor. Energ Fuel 28: 7721-7725 (2014).

49. Dias FM, Bundaleska N, Henriques J, Tatarova E and Ferreira CM, Microwave plasma torches used for hydrogen production. 4th International Workshop \& Summer School on Plasma Physics 2010516 (2014).

50. Wang YF, You YS, Tsai CH and Wang LC, Production of hydrogen by plasma-reforming of methanol. Int J Hydrogen Energ 35: 9637-9640 (2010).

51. Khani MR, Guy ED, Gharibi M, Shahabi SS, Khosravi A, Norouzi AA and Shokri B, The effects of microwave plasma torch on the cracking of Pyrolysis Fuel Oil feedstock. Chem Eng J 237: 169-175 (2014).

52. Tatarova E, Bundaleska N, Dias FM, Tsyganov D, Saavedra R and Ferreira CM, Hydrogen production from alcohol reforming in a microwave 'tornado'-type plasma. Plasma Sources Sci T 22 (2013).

53. Radoiu MT, Studies on atmospheric plasma abatement of PFCs. Radiat Phys Chem 69: 113120 (2004).

54. Terry LW, System to continuously produce carbon fiber via microwave assisted plasma processing. US Patent: 8679592 (2014).

55. Schlemm H, Mai A, Roth S, Roth D, Baumgartner KM and Muegge H, Industrial large scale silicon nitride deposition on photovoltaic cells with linear microwave plasma sources. Surf Coat Tech 174: 208-211 (2003).

56. Adtec, SteriPlas, http://www.adtecplasma.com/products.html\#home. (accessed 25-09-2016).

57. Dischler B and Wild C, Synthetic Diamond: Manufacturing and Applications. Springer-Verlag (1998).

58. Rummel $\mathrm{P}$ and Grotjohn TA, Methods for modeling microwave plasma system stability. Journal of Vacuum Science \& Technology a-Vacuum Surfaces and Films 20: 536-543 (2002).

59. Samukawa S, Hori M, Rauf S, Tachibana K, Bruggeman P, Kroesen G, Whitehead JC, Murphy AB, Gutsol AF, Starikovskaia S, Kortshagen U, Boeuf JP, Sommerer TJ, Kushner MJ, Czarnetzki U and Mason N, The 2012 Plasma Roadmap. J Phys D Appl Phys 45 (2012).

60. Bogdankevich IL, Loza OT and Pavlov DA, Frequency stability in plasma relativistic microwave oscillators. Tech Phys Lett+ 33: 629-631 (2007).

61. Bundaleska N, Tsyganov D, Tatarova E, Dias FM and Ferreira CM, Steam reforming of ethanol into hydrogen-rich gas using microwave Ar/water "tornado" - Type plasma. Int J Hydrogen Energ 39: 5663-5670 (2014).

62. Reszke E, Split energy delivery to material heating at RF and microwave frequencies. Prz Elektrotechniczn 88: 354-358 (2012).

63. Willis KP, Osada S and Willerton KL, Plasma Gasification: Lessons Learned at EcoValley WTE Facility. 18th Annual North American Waste-to-Energy Conference (2010).

64. Kiss AA, Pragt JJ, Vos HJ, Bargeman G and de Groot MT, Novel efficient process for methanol synthesis by CO2 hydrogenation. Chem Eng J 284: 260-269 (2016).

65. Schluter $\mathrm{H}$ and Shivarova A, Advanced technologies based on wave and beam generated plasmas, in NATO ASI Series. Springer Netherlands (1999).

66. Metaxas AC and Meredith RJ, Industrial Microwave Heating, Volume 1. IEE Power Engineering Series 4 (1993).

67. ATKearney, Chemical Industry Vision 2030: A European Perspective, https://www.atkearney.n1/documents/10192/536196/Chemical+Industry+Vision+2030+A+Eur opean+Perspective.pdf/7178b150-22d9-4b50-9125-1f1b3a9361ef. (accessed 05-01-2017). 
1 68. Hessel V, Cravotto G, Fitzpatrick P, Patil BS, Lang J and Bonrath W, Industrial applications 2 of plasma, microwave and ultrasound techniques: Nitrogen-fixation and hydrogenation 3 reactions. Chem Eng Process 71: 19-30 (2013). 